\title{
Enseñanza de la asignatura de Urgencias en universidades mexicanas
}

\author{
Ana P. Pérez-Ortega ${ }^{1}$, Haidee I. Uriostegui-Santiago ${ }^{2}$ y Alejandro Villatoro-Martínez ${ }^{3^{*}}$ \\ ${ }^{1}$ Servicio de Urgencias, Unidad de Medicina Familiar 26, Hospital General de Zona, Instituto Mexicano del Seguro Social, Tala, Jalisco; ${ }^{2}$ Jefatura \\ de Medicina familiar, Unidad de Medicina Familiar 20, Instituto Mexicano del Seguro Social, Cuernavaca, Mor.; ${ }^{3}$ Coordinación de programas \\ médicos/Coordinación de investigación en salud, Centro Médico Nacional Siglo XXI, Instituto Mexicano del Seguro Social, Ciudad de México, \\ Méx. México
}

\section{Resumen}

Introducción: Los servicios de urgencias en México concentran el $80 \%$ de los ingresos hospitalarios. Siendo una necesidad formar personal médico capacitado para proporcionar atención médica de urgencia, nuestra pregunta fue ¿Cuál es el panorama actual de esta enseñanza en México? Objetivo: Analizar las características del proceso educativo de la enseñanza de las urgencias en las facultades de medicina de las universidades mexicanas. Método: Se realizó y envió por correo electrónico una encuesta descriptiva, observacional y transversal a universidades mexicanas sobre la enseñanza de Medicina de Urgencia (MU) en pregrado y posgrado. Conclusiones: Como materia no ha sido reconocida en todas las universidades dentro del plan de estudios de la carrera de médico general y no se le ha otorgado relevancia a la materia. Se presentan recomendaciones basadas en el estudio para las universidades para mejorar la enseñanza sobre MU.

Palabras clave: Urgencias médicas. Enseñanza. Universidades. México.

\section{Teaching of the subject of Emergency Medicine in Mexican universities}

\begin{abstract}
Introduction: emergency services in Mexico account for $80 \%$ of hospital admissions. In our country there is a need to train medical personnel to provide emergency medical care. Objective: to analyze the characteristics of the teaching of urgencies and emergencies in the medical faculties of the Mexican universities. Method: a transversal, descriptive and observational survey was carried out, sent by email to Mexican universities questioning about the teaching of emergency Medicine (EM). Results: it was Determined that in $32 \%$ of the faculties that Teach the Subject, Seniority is less than 5 years, $76 \%$ is compulsory, 35\% of the Universities Teach it in Eighth semester, $85 \%$ have a curriculum, $88 \%$ have a Practical Subject, as well as in hospital 88\%; it is taught by an Emergency doctor in 70\% of the cases, $12 \%$ by an internist and $12 \%$ by a Surgeon; as a requirement for Leaving only 8 Universities Ask for a CPR, ACLS or ATLS course, only 7 Universities complete more than 15 Recommended Subject areas. Conclusions: The subject of medical emergencies has not been recognized in all universities within the curriculum of the general practitioner's degree; it has not been given relevance to the subject.
\end{abstract}

Key words: Emergency medicine. Teaching. Universities. Mexico.

\section{Correspondencia:}

*Alejandro Villatoro-Martínez

E-mail: alexvillatorom @gmail.com
Fecha de recepción: 18-08-2020

Fecha de aceptación: 08-03-2021 DOI: 10.24875/REIE.20000037
Disponible en internet: 30-07-2021

Rev Educ Investig Emer. 2021;3(2):45-51 www.medicinadeemergencias.com 2604-6520 @ 2021 Sociedad Mexicana de Medicina de Emergencias, AC. Publicado por Permanyer México SA de CV. Este es un artículo open access bajo la licencia CC BY-NC-ND (http://creativecommons.org/licenses/by-nc-nd/4.0/). 


\section{Introducción}

Los servicios de urgencias de adultos y pediatría en México concentran el $80 \%$ de los ingresos hospitalarios $^{1}$. La medicina de urgencias (MU) en el país surge posterior al sismo de 1985, en donde varias personas perdieron la vida por causas secundarias a la falta de infraestructura y preparación de los servicios atención en salud ${ }^{1-3}$. Por ello se creó la especialidad de urgencias medicoquirúrgicas, con sede en el Hospital General Balbuena, surgiendo como especialidad en México y Latinoamérica'. Como toda especialidad, considera importante formar personal médico capacitado que proporcione atención médica de urgencia adecuada ante procesos agudos y crónicos agudizados y desastres $^{4}$. Actualmente persiste la escasez de estos especialistas; en un artículo reciente se indica que hay 76.71 neonatólogos por cada 100,000 habitantes, 49.71 pediatras; 8.09 cirujanos generales y solo 1.25 especialistas en $\mathrm{MU}^{5}$. La gran mayoría de las instituciones de educación superior en medicina respaldan como especialidad a la MU, pero su enseñanza en futuros egresados no se ha difundido adecuadamente en nuestro país ${ }^{6-9}$.

En 1991 se consiguió que la especialidad se ofreciera dentro del catálogo de especialidades del Instituto Mexicano del Seguro Social, aumentando el número de plazas de la especialidad. En 1998 se dio el aval académico universitario por el Instituto Politécnico Nacional, además de implementar la asignatura dentro de la carrera, aumentando gracias a ello el conocimiento y habilidades en urgencias del estudiante de pregrado'. En la actualidad, las universidades en nuestro país consideran que para la adecuada formación de sus egresados se contemple un aprendizaje ético y que desarrolle habilidades para aumentar su capacidad resolutiva, por lo anterior varias de ellas han incluido la formación del área de urgencias dentro de las asignaturas del currículum de la carrera, con la finalidad de que cuando el alumno egrese, tenga los conocimientos necesarios para desarrollar toda su capacidad, habilidad y conocimientos en cualquier situación de urgencia ${ }^{10}$.

Un punto que considerar dentro de la educación en MU es que no existen recomendaciones de parte de las universidades, academias y asociaciones del país ${ }^{11}$ sobre cómo enseñar, desarrollar y mejorar la atención de la MU. La Sociedad Española de Medicina de Urgencias y Emergencias (SEMES) sugiere las siguientes recomendaciones $^{6}$ : que sea una asignatura obligatoria, impartida entre el tercer y cuarto año de la carrera, con clases teóricas y práctica intrahospitalaria de 1-2 meses por un servicio de urgencias en todas sus fases, y que sea considerada antes del internado.

El área de urgencias es el inicio de la atención de muchos pacientes, permitiendo su clasificación y ubicación final. Por ello dentro de los servicios de urgencias no solo hay urgenciólogos u otros especialistas, sino también internos de pregrado que acaban de terminar su formación académica, por lo que la enseñanza de urgencias médicas en la facultad es de gran relevancia ${ }^{11}$.

Consideramos que la enseñanza sobre MU en universidades en México ha tenido deficiencias, sobre todo en estructura, programa y formación de médicos; sin una unión entre asociaciones de MU y universidades, la enseñanza es parcial. De ahí nace nuestra pregunta de investigación: ¿Cuál es el panorama actual de esta enseñanza en México?

\section{Objetivo}

Analizar las características del proceso educativo de la enseñanza de las urgencias en las facultades de medicina de las universidades mexicanas.

\section{Método}

Se analizó la lista de asociados a la Asociación Mexicana de Facultades y Escuelas de Medicina, A.C. (AMFEM), excluyendo las escuelas sin acreditación actualizada (Tabla 1). Se consiguió el listado de correos electrónicos y teléfono de cada universidad para completar una agenda. El trabajo fue realizado en el año 2015.

Se creó un cuestionario de recolección de datos basado en el trabajo previo de Coll Vinent, et al. ${ }^{6}$ para determinar las características de la impartición de la materia en las universidades. El cuestionario valoraba varios tópicos: impartición, horas a la semana, materia práctica, quién la imparte, cursos relacionados y temas básicos dentro de la materia. El estudio es un trabajo exploratorio, pero fue ingresado al comité de investigación y ética del hospital (hospital de tercer nivel), que es sede de la especialidad del Instituto Mexicano del Seguro Social, siendo aceptado, al igual que en el comité tutelar de la especialidad de la Sección de Posgrado e Investigación de la Escuela Superior de Medicina del Instituto Politécnico Nacional.

Una vez realizado el cuestionario con respecto a su programa académico, se envió por correo electrónico a las 53 universidades para que fuera autocompletado, para explorar con qué criterios se imparte la materia en MU. Se dio un plazo de 10 días para 
Tabla 1. Universidades acreditadas por la Asociación Mexicana de Facultades y Escuelas de Medicina, A.C. (AMFEM)

\begin{tabular}{|c|c|c|}
\hline Número & Universidad o institución & Localidad \\
\hline 1 & Benemérita Universidad Autónoma de Puebla & Puebla \\
\hline 2 & Instituto de Estudios Superiores de Chiapas (Tapachula) & Chiapas \\
\hline 3 & Instituto de Estudios Superiores de Chiapas (Tuxtla) & Chiapas \\
\hline 4 & Instituto Politécnico Nacional & Ciudad de México \\
\hline 5 & Instituto Superiores de Monterrey Ignacio Santos & Monterrey \\
\hline 6 & Universidad Benito Juárez de Oaxaca & Oaxaca \\
\hline 7 & Universidad La Salle & Ciudad de México \\
\hline 8 & Universidad Anáhuac & Ciudad de México \\
\hline 9 & Universidad Autónoma Metropolitana & Ciudad de México \\
\hline 10 & Universidad Autónoma de Baja California & Baja California \\
\hline 11 & Universidad Autónoma de Campeche & Campeche \\
\hline 12 & Universidad Autónoma de Chiapas & Chiapas \\
\hline 13 & Universidad Autónoma de Coahuila plantel Saltillo & Coahuila \\
\hline 14 & Universidad Autónoma de Coahuila plantel Torreón & Coahuila \\
\hline 15 & Universidad Autónoma de Colima & Colima \\
\hline 16 & Universidad Autónoma de Guanajuato & Guanajuato \\
\hline 17 & Universidad Autónoma de México & Ciudad de México \\
\hline 18 & Universidad Autónoma de Morelos & Morelos \\
\hline 19 & Universidad Autónoma de Nayarit & Nayarit \\
\hline 20 & Universidad Autónoma de Querétaro & Querétaro \\
\hline 21 & Universidad Autónoma de San Luis Potosí & San Luis Potosí \\
\hline 22 & Universidad Autónoma de Sinaloa & Sinaloa \\
\hline 23 & Universidad Autónoma de Tlaxcala & Tlaxcala \\
\hline 24 & Universidad Autónoma de Yucatán & Yucatán \\
\hline 25 & Universidad Autónoma del Estado de Hidalgo & Hidalgo \\
\hline 26 & Universidad de Aguascalientes & Aguascalientes \\
\hline 27 & Universidad de Chihuahua & Chihuahua \\
\hline 28 & Universidad de Guadalajara (Centro Univer. de la Costa) & Jalisco \\
\hline 29 & Universidad de Guerrero & Guerrero \\
\hline 30 & Universidad de las Américas Puebla & Puebla \\
\hline 31 & Universidad de Monterrey & Monterrey \\
\hline 32 & Universidad de Nuevo León & Nuevo León \\
\hline 33 & Universidad de Quintana Roo & Quintana Roo \\
\hline 34 & Universidad de Quiroga & Ciudad de México \\
\hline 35 & Universidad de Sonora & Ciudad de México \\
\hline 36 & Universidad de Tepeaca & Ciudad de México \\
\hline
\end{tabular}

esperar respuesta. Si ello no ocurría se les realizaba la entrevista de manera telefónica. Los datos se analizaron y recopilaron en tablas, realizando los gráficos correspondientes. Se realizó el análisis descriptivo de los datos.

El protocolo de investigación fue aceptado por el comité de ética e investigación del hospital y en su momento por el posgrado universitario de la especialidad.

\section{Resultados}

De las 53 universidades acreditadas, solo 38 universidades respondieron el cuestionario enviado en forma electrónica. Completado el primer periodo, todavía había 15 universidades que no habían respondido. Se terminó la encuesta mediante llamadas telefónicas a los coordinadores de las facultades de medicina faltantes; a pesar de lo anterior no se contactó con los coordinadores de 9 universidades, teniendo que excluirlos del estudio. Se presentan los datos de 44 universidades y se encontró que solo 17 (38.63\%) imparten la materia de urgencias médicas como asignatura (Fig. 1) y a nivel de pregrado; en solo una universidad la imparten en posgrado como aval universitario (Fig. 2).

La antigüedad en que se imparte la materia en las universidades se encuentra que en la mayoría es menor a 5 años, solo algunas la han impartido por más de 10 años (Tabla 2). Se observó que en el $76 \%$ de las universidades la materia es obligatoria. El semestre en donde la mayoría de las universidades la imparte es en octavo semestre, teniendo en su mayoría un temario formal, excepto en dos universidades.

En la mayoría de las universidades la asignatura es teórico-práctica (en universidad e intrahospitalaria). La 


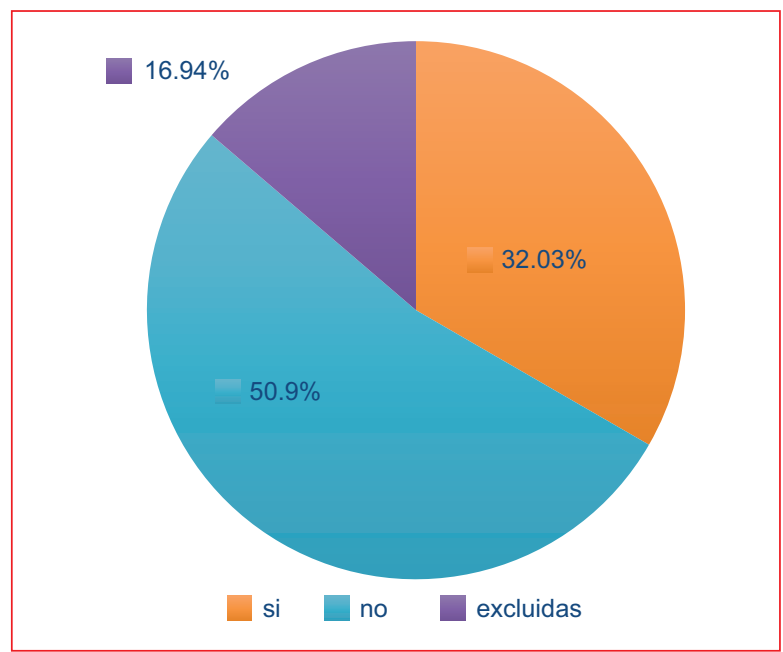

Figura 1. Porcentaje de universidades que imparten la materia de urgencias médicas.

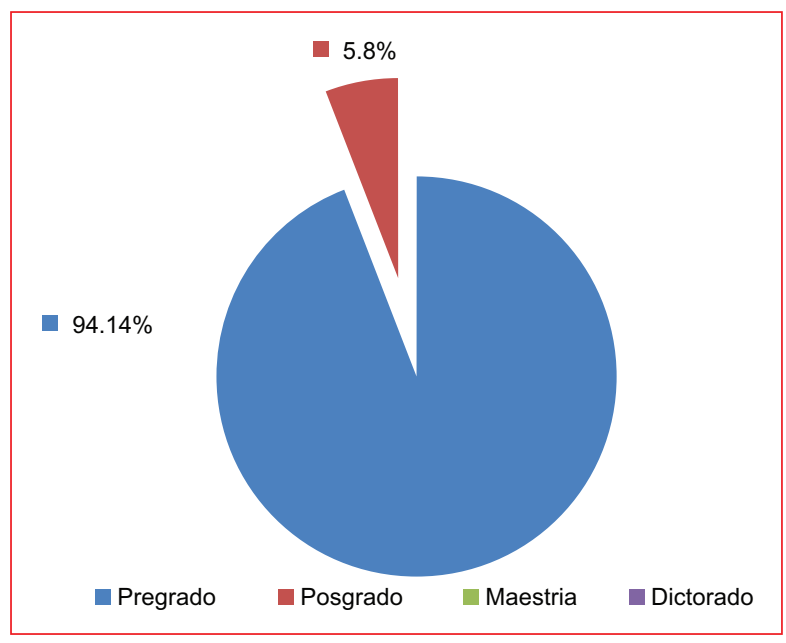

Figura 2. Porcentaje de impartición de la asignatura por grado.

materia práctica intrahospitalaria se considera relevante, impartiéndose en el $88 \%$ de las universidades. Es impartida por urgenciólogos en el $70 \%$ de las universidades (Fig. 3), pero todavía es impartida por profesores de otras especialidades: un $12 \%$ por cirujanos, un $6 \%$ por internista y un $12 \%$ por otros especialistas.

Para el ingreso a la carrera no se solicita ningún curso de reanimación (BLS, basic life support) y al egreso solo en dos universidades piden cursos certificados como ATLS (apoyo vital avanzado en trauma) y ACLS (soporte vital cardiovascular avanzado); en seis solicitan cursos de reanimación básica, pero de forma monográfica.

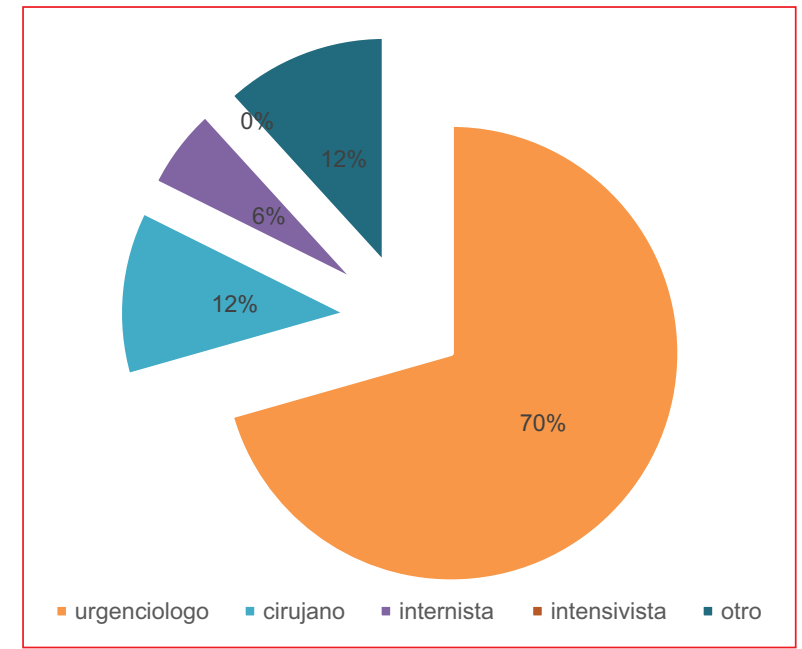

Figura 3. Materia de urgencias médicas impartida por el especialista.

Tabla 2. Años de antigüedad de impartición de la materia

\begin{tabular}{|l|c|}
\hline Universidad & $\begin{array}{c}\text { Año de inicio de } \\
\text { impartición }\end{array}$ \\
\hline Universidad Autónoma de Baja California & 2000 \\
Universidad Autónoma de San Luis Potosí & 2007 \\
Universidad de Monterrey & 1969 \\
Universidad Juárez de Durango campus & 1974 \\
Gómez Palacio & \\
Universidad de Sonora & 2000 \\
Instituto Politécnico Nacional & 2003 \\
Universidad de Guadalajara (Centro Univer. & 1999 \\
de la Costa) & 2001 \\
Universidad de Westhill & 1996 \\
Universidad Panamericana & 1997 \\
Universidad Autónoma de México & en proceso \\
Universidad Justo Sierra & no especificado \\
Universidad Montemorelos & 1999 \\
Universidad del Sureste & 2009 \\
Universidad Autónoma de Colima & 2002 \\
Universidad Autónoma de Yucatán & 2004 \\
Universidad Autónoma de Querétaro & 2000 \\
Universidad de Las Américas Puebla &
\end{tabular}

Sobre temas monográficos seleccionados como indispensables para la formación en MU de los estudiantes, la mayoría de las universidades completan los 19 temas y como mínimo cuentan con 13 temas, de lo básico hasta patologías específicas.

\section{Discusión}

A pesar que las urgencias médicas son eventos que ocurren en numerosos procesos patológicos, durante el estudio de cada una de las diferentes materias en 
la carrera son abordados como parte de esa especialidad, no como el conjunto de conocimientos que agrupa todas aquellas situaciones clínicas que van a demandar con carácter urgente que es la $\mathrm{MU}^{12}$. Pero la escasez de medios de urgencias $^{13}$ hace importante la frecuencia con que a los recién egresados se les ofrece trabajar de médico de urgencias, donde atienden con pánico $o$ acuden atropelladamente al servicio urgencia de un hospital, donde «hicieron una práctica» o conocen a un médico, que les permita "doctorarse" en MU durante el fin de semana, para asumir el lunes, con responsabilidad plena, la función de médico de urgencia $^{12}$. Lo anterior ocurre debido a que la enseñanza de MU en facultades y escuelas de medicina mexicanas todavía es baja, en nuestro estudio se imparte en casi el $40 \%$ de las universidades encuestadas, 10 que permite reflexionar que la especialidad todavía no tiene un papel preponderante como otras asignaturas, además de que solo se imparte en pregrado en el $90 \%$, el restante $10 \%$ sugiere que pocas universidades avalan la materia de urgencias como especialidad, demeritando su importancia', a pesar de que es una especialidad integral, que permite mejorar la atención a los pacientes que acuden a este servicio ${ }^{13}$.

Con respecto a la antigüedad de impartición, en más del $50 \%$ es de cinco años o menos, además, en algunos casos, como en la universidad Justo Sierra, ya se contempla dentro del programa de estudios, pero prospectivamente. Lo que sugiere que las universidades particulares están incorporando la materia de UM más rápido dentro de su enseñanza ${ }^{2,13}$.

La forma de impartición demuestra su importancia para la universidad, ya que al ser opcional permite al estudiante solicitar otra materia. Consideramos que es aceptable que el $76 \%$ la consideren obligatoria, lo cual es benéfico para su formación. La recomendación de la SEMES es que sea de forma obligatoria. En México no hay pronunciamientos ni publicaciones de las asociaciones médicas al respecto ${ }^{14-17}$.

Consideramos oportuno que la materia de MU sea impartida en el tercer o cuarto año de la carrera, para que los estudiantes tengan conocimientos básicos de anatomía, fisiología y patología ${ }^{18,19}$. Esto se cumple en seis universidades. La recomendación es que sea entre el quinto y sexto semestre dentro de las materias clínicas. El contar con una materia práctica permite desarrollar habilidades y conocimientos que se aplicaron en la atención de los pacientes. Quince facultades imparten la materia de manera teórica en la escuela, y posteriormente intrahospitalaria, el $88 \%$ de las facultades la imparten en un contexto intrahospitalario ${ }^{12,20,21}$.
Conocer quién imparte la materia es importante, ya que determina el punto de vista y visión del docente y hay que tener en cuenta que la relevancia de prioridades ante una urgencia cambia entre especialistas. En nuestra muestra predominaron los urgenciólogos en un $70 \%$, considerándose lo adecuado, pero en el $30 \%$ restante había internistas, cirujanos e intensivistas ${ }^{3}$. Lo anterior desafortunadamente ocurre también en la Facultad de Medicina de la Universidad Nacional Autónoma de México, donde el Programa Único de Especializaciones Médicas (PUEM) fue diseñado en un $66 \%$ por especialistas que no son urgenciólogos ${ }^{22}$.

Solo dos universidades ponen como requisito tener un curso de BLS al egreso de la licenciatura, lo anterior se debe difundir a todas las universidades. Además, antes del egreso de la universidad los estudiantes deberían de contar con la certificación en ATLS y ACLS. Como recomendación del grupo de estudio es que los estudiantes conozcan y apliquen el BLS desde su ingreso a la carrera, mismo que idealmente se debería de tener vigente durante la carrera, ya que mejorará la atención de los pacientes graves ${ }^{21-24}$.

Desafortunadamente, las sociedades y asociaciones mexicanas de emergencias no han realizado posicionamientos o recomendaciones de la necesidad de formación universitaria. Otra recomendación que sugiere este grupo es que existan grupos de trabajo entre asociaciones y universidades, para lograr mayor avance en la enseñanza ${ }^{25-27}$. De hecho se están diseñando nuevos modelos de enseñanza en MU, como es la rotación de residentes en ambulancias y helicópteros de traslado ${ }^{28}$. Aun cuando le ganamos a España a implementar la especialidad dentro del país, y por ende la aceptación en universidades e instituciones, los avances logrados por la SEMES como la aceptación de la revista, la difusión de la especialidad en ese país y en el mundo son mucho más relevantes $24,29-32$.

Limitaciones del estudio: como todo estudio exploratorio (inicial), no contamos con estudios previos para compararlo. Además, el cuestionario siguió las pautas realizadas por Coll Vinent, et al., por lo que no está todavía validado para el país por las características del mismo (cuestionario autocompletado y contacto telefónico), no tenemos la información de primera mano y podría haber sesgos en las respuestas y, finalmente, el desfase en el tiempo en el que se realizó el estudio. No obstante, consideramos que los resultados son de utilidad para tener un primer acercamiento de cómo se enseña la MU en México.

Como resultado de futuras investigaciones se pueden diseñar programas de MU que sean adecuados para cada entidad y para la realidad del país, se debe 
Tabla 3. Recomendaciones para la carrera La materia debe ser incluida dentro del mapa curricular de la
carrera de médico

Impartida por un médico especialista en medicina de urgencias

Asignatura obligatoria, con $4 \mathrm{~h}$ semanales (1 h práctica).

Impartirla entre el $7 .^{\circ}$ y $8 .^{\circ}$ semestre

Previa al internado, guardias 2 a 4 h, 2 guardias semanales. 20 guardias, 10 servicio urgencias (área de choque, observación, consultorios), 5 tococirugía y 5 urgencias traumatológicas

Temario sugerido básico para la materia (semiología, diagnóstico y tratamiento inicial)

1. Primeros auxilios

2. Estabilización paciente en urgencias

3. Cefaleas

4. Vía aérea en urgencias

5. Arritmias

6. Dolor torácico y abdominal

7. Diagnóstico y tratamiento de la disnea

8. Diagnóstico y tratamiento del edema agudo de pulmonar

9. Diagnóstico y tratamiento de la neumonía

10. Crisis hipertensiva

11. Insuficiencia cardiaca

12. Síndromes coronarios

13. Hemorragia de tubo digestivo alto y bajo

14. Insuficiencia renal aguda y crónica

15. Fiebre en estudio

16. Manejo de las urgencias en diabetes mellitus

17. Cirrosis hepática

18. Intoxicaciones

Requisito para presentar examen profesional, tener mínimo BLS, e incluso ATLS y ACLS.

BLS: basic life support, ATLS: apoyo vital avanzado en trauma; ACLS: soporte vital cardiovascular avanzado.

Coll Vinent B, Sánchez M, Nogué R, Miró 0. La enseñanza de la Medicina de Urgencias y Emergencias en las facultades de medicina españolas: situación actual. Emergencias 2010;22:21-27.

enseñar a los estudiantes que la especialidad es integral y que permite aplicar los conocimientos de todas las especialidades, que la MU no solo es trauma, por lo que en ambas se deberán de hacer encuestas para considerar qué piensan los estudiantes de medicina de la MU. Es preciso tener un cuerpo de profesores de MU que sea fuerte, posicionado en lugares que permitan hacer crecer la espacialidad y publicar mucho más a nivel nacional e internacional.

\section{Conclusiones}

En nuestro país la MU ha tenido avances, pero falta consolidar su posición en la enseñanza de médicos generales en cursos de pregrado y posgrado en medicina.

Se deben realizar más estudios sobre este tópico, para difundir más la materia y la especialidad, ya que es importante que los médicos conozcan el diagnóstico y manejo adecuado de las urgencias médicas en bien de los pacientes y de ellos mismos como profesionales. Difundir la enseñanza de la MU permite exponer las actualizaciones y apoyo a la educación de calidad. Debe haber mayor comunicación entre asociaciones de urgencias y universidades, lo que permitirá ampliar la visión de la enseñanza de MU actual. Como un extra de la presente investigación, en la tabla 3 se sugieren recomendaciones basadas en la SEMES.

\section{Financiamiento}

La presente investigación no ha recibido ninguna beca especifica de agencias de los sectores público, comercial o sin ánimo de lucro.

\section{Conflicto de intereses}

Los autores declaran no tener conflicto de intereses.

\section{Responsabilidades éticas}

Protección de personas y animales. Los autores declaran que para esta investigación no se han realizado experimentos en seres humanos ni en animales.

Confidencialidad de los datos. Los autores declaran que han seguido los protocolos de su centro de trabajo sobre la publicación de datos de pacientes.

Derecho a la privacidad y consentimiento informado. Los autores declaran que en este artículo no aparecen datos de pacientes.

\section{Bibliografía}

1. Rocha Luna JM. Historia de la medicina de urgencia en México. Arch Med Urg Méx. 2009;1:4-11.

2. Fernández Pérez JA. Las carreras de Medicina en México. Perfiles Educativos 1996; XVIII 73.

3. Villatoro Martínez A. Educación en medicina de urgencia. Arch Med Urg Méx. 2012;4:3-5.

4. Pradere-Pensado JC, Chao-González S, García Gómez A, GutiérrezGutiérrez L. Consideraciones éticas sobre la atención médica en situaciones de desastre. MEDISAN 2012;16(8):1296-306.

5. Heinze-Martin G, Olmedo-Canchola VH, Bazán-Miranda G, BernardFuentes NA, Guízar-Sánchez DP. Los médicos especialistas en México. Gac Méd Méx. 2018;154:342-51.

6. Coll Vinent B, Sánchez M, Nogué R, Miró O. La enseñanza de la medicina de urgencias y emergencias en las facultades de medicina españolas: situación actual. Emergencias. 2010;22:21-7.

7. Villatoro Martínez A. Definiciones básicas en medicina de urgencia [Internet]. Reeme.arizona.edu; 2005. Disponible en: https://silo.tips/download/definiciones-basicas-en-medicina-de-urgencia

8. Reta de Rosas AM, López MJ. Competencias médicas y su evaluación al egreso de la carrera de medicina en la Universidad Nacional de Cuyo (Argentina). Educación Médica. 2006;9:75-83.

9. Declaración de principios sobre la Medicina de Urgencia en Europa. Sociedad Europea de Medicina de Urgencia (EuSEM). Septiembre de 2007.

10. Loria Castellanos J, Márquez Ávila G, Valladares Aranda MA. Procedimiento realizados por residentes de la especialidad de urgencias en una sede de México. Gac Méd Méx. 2010;146:103-4.

11. Rocha Luna JM. 25 años de la medicina de urgencia. Los retos... ¿siguen siendo los mismos? Arch Med Urg Méx. 2011;3(1):3-5. 
12. Juárez Alonso S. La enseñanza de la medicina de urgencia, una asignatura pendiente. Emergencias. 1999;11:91-2

13. Tudela $P$, Mòdol JM. La saturación en los servicios de urgencias hospitalarios. Emergencias. 2015;27:113-20.

14. Castillo de los Santos R. Medicina de urgencias: una especialidad nueva para el gremio médico militar, pero vital para el servicio de sanidad militar. Rev Sanid Milit Méx. 2017;71:290-9.

15. Urrutia Aguilar ME, Guevara Guzmán R. Estrategias docentes en el primer año de la carrera de médico cirujano y nivel de aprovechamiento académico. Inv Ed Med. 2013;2:77-81.

16. Coll Vinent B, Torres S, Sánchez A. Catalan medical students' predisposition to specialize in emergency medicine. Emergencias. 2010;22:15-20.

17. Seefeld A. Lessons learned from working in emergency departments in Cape Town, South Africa: a final-year medical student's perspective. S Afr Med J. 2007;97:21-8.

18. Geary U, Kennedy U. Toma de decisiones clínicas en medicina de urgencias y emergencias. Emergencias. 2010;22:56-60.

19. Juárez Alonso S. Cuando llega la jubilación. Rev Clín Esp 2011;211:260-4

20. Cuacuas Cano V. La certificación en medicina de urgencias. Arch Med Urg Méx. 2011;3:47-8.

21. Rocha Luna JM. La educación en medicina de urgencia. En: Loria Castellanos J. Medicina de urgencia en México. Tres décadas. Logros y retos. 1. ${ }^{\text {a }}$ edición. México: Ediciones Berit; 2017. pp. 47-57.

22. Trujillo Galván FD, Calderón Díaz $R$, Clemente Herrera A López Bazán TF, Montiel Falcón HM, Baltazar Olivar VC, et al. Plan único de especializaciones médicas. México: Facultad de Medicina, Universidad Nacional Autónoma de México; 2015
23. Ayuso F, Nogué R, Coll-Vinent B. Docencia en medicina de urgencias y emergencias. An Sist Sanit Navar. 2010;33:203-13.

24. Mejía C, Quesada Osoria C, Moras Ventocilla C. Nivel de conocimientos sobre emergencias médicas en estudiantes de medicina de universidades peruanas. Rev Peru Med Exp Salud Pública. 2011;28:202-9.

25. Russi C, Hamilton GC. A case for emergency medicine in the undergraduate medical school curriculum. Acad Emerg Med. 2005;12:994-8.

26. Polglase R, Parish D, Buckley RL. Problem-based ACLS instruction: a model approach for undergraduate emergency medical education. Ann Emerg Med. 1989;18:997-1000.

27. Creutzfeldt J, Hedman L, Medin C. Implementing virtual worlds for systematic training of prehospital CPR in medical school. Stud Health Technol Inform. 2007;125:82-4.

28. Wald DA, Lin M, Manthey DE. Emergency medicine in the medical school curriculum. Acad Emerg Med. 2010;17:S26-S30.

29. Mancera M, Kraut A, Gussick M, Lohmeier M. A model curriculum for an emergency medical services EMS rotation for emergency medicine residents. J Educ Teach Emerg Med. 2018;3:23-8.

30. Razzak J, Ahmed A. Perceived need for emergency medicine training in Pakistan: a survey of medical education leadership. Emerg Med Australas. 2009;21(2):143-6.

31. Weller J, Robinson B, Larsen P, Caldwell C. Simulation-based training to improve acute care skills in medical undergraduates. N Z Med J. 2004:117(1204):U1119.

32. Khandelwal S, Way DP, Wald DA. State of undergraduate education in emergency medicine: a national survey of clerkship directors. Acad Emerg Med. 2014;21:92-5. 